\title{
Impact of Serum Cytokine Levels on EEG-Measured Arousal Regulation in Patients with Major Depressive Disorder and Healthy Controls
}

\author{
Frank M. Schmidt ${ }^{a}$ Annika Pschiebla Christian Sander ${ }^{a}{ }^{b}$ Kenneth C. Kirkby ${ }^{d}$ \\ Julia Thormann ${ }^{a, b}$ Juliane Minkwitz ${ }^{a, b}$ Tobias Chittka ${ }^{a, b} \quad$ Julia Weschenfelder ${ }^{a}$ \\ Lesca M. Holdt ${ }^{c}$ Daniel Teupser ${ }^{c}$ Ulrich Hegerl ${ }^{a}$ b Hubertus Himmerich ${ }^{a}$ b \\ ${ }^{a}$ Department of Psychiatry and Psychotherapy, University Hospital Leipzig, and ${ }^{\mathrm{b}}$ Leipzig University Medical Center, \\ Integrated Research and Treatment Center Adiposity Diseases (IFB), Leipzig, and ' Institute of Laboratory Medicine,

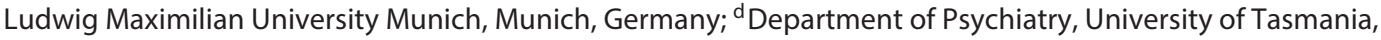 \\ Hobart, Tas., Australia
}

\section{Key Words}

Cytokines · Depression - Immunity · Resting-state EEG .

Sleep-wake regulation - Vigilance regulation $\cdot$ Brain arousal

\begin{abstract}
Background: In major depressive disorder (MDD), findings include hyperstable regulation of brain arousal measured by electroencephalography (EEG) vigilance analysis and alterations in serum levels of cytokines. It is also known that cytokines affect sleep-wake regulation. This study investigated the relationship between cytokines and EEG vigilance in participants with MDD and nondepressed controls, and the influence of cytokines on differences in vigilance between the two groups. Methods: In 60 patients with MDD and 129 controls, 15-min resting-state EEG recordings were performed and vigilance was automatically assessed with the VIGALL 2.0 (Vigilance Algorithm Leipzig). Serum levels of the wakefulnesspromoting cytokines interleukin (IL)-4, IL-10, IL-13 and somnogenic cytokines tumor necrosis factor- $\alpha$, interferon- $\gamma$ and IL-2 were measured prior to the EEG. Results: Summed wakefulness-promoting cytokines, but not somnogenic cytokines,
\end{abstract}

were significantly associated with the time course of EEG vigilance in the MDD group only. In both groups, IL-13 was significantly associated with the course of EEG vigilance. In MDD compared to controls, a hyperstable EEG vigilance regulation was found, significant for group and group $\times$ time course interaction. After controlling for wakefulness-promoting cytokines, differences in vigilance regulation between groups remained significant. Conclusions: The present study demonstrated a relationship between wakefulness-promoting cytokines and objectively measured EEG vigilance as an indicator for brain arousal. Altered brain arousal regulation in MDD gives support for future evaluation of vigilance measures as a biomarker in MDD. Since interactions between cytokines and EEG vigilance only moderately differed between the groups and cytokine levels could not explain the group differences in EEG vigilance regulation, cytokines and brain arousal regulation are likely to be associated with MDD in independent ways.

(c) 2016 S. Karger AG, Basel

Frank M. Schmidt and Annika Pschiebl contributed equally to this work.

\section{KARGER}

E-Mail karger@karger.com

www.karger.com/nps
(C) 2016 S. Karger AG, Basel

0302-282X/16/0731-0001\$39.50/0
Dr. med. Frank M. Schmidt

Department of Psychiatry and Psychotherapy, University Hospital Leipzig Semmelweisstrasse 10

DE-04103 Leipzig (Germany)

E-Mail Frank.Schmidt2@medizin.uni-leipzig.de 


\section{Introduction}

The majority of patients suffering from major depressive disorder (MDD) not only exhibit core symptoms of MDD such as depressed mood, reduced drive and loss of interest, but also disturbances pointing to a preponderance of wakefulness-promoting over sleep-promoting (somnogenic) brain mechanisms. Among these symptoms are prolonged sleep onset latencies, impaired sleep maintenance, inner tension and difficulty relaxing. Within the arousal model of affective disorders these symptoms are associated with a high and hyperstable regulation of vigilance, which is postulated to play an important pathogenetic role [1]. Within this model, symptoms of sensation avoidance and social withdrawal are interpreted as an autoregulatory reaction of the organism to this state of high brain arousal: the organism aims to avoid any further arousal-increasing sensory or social stimulation. Using resting-state EEG (electroencephalography) recordings, a replicated finding is that patients with MDD compared to healthy controls show hyperstable EEG vigilance regulation characterized by fewer and later transitions to EEG signs of drowsiness or sleep onset $[2,3]$.

Cytokines, transmitters of the immune system, have been shown to be involved in both wakefulness regulation and the pathogenesis of depression. They influence several neurotransmitter systems such as the serotonin, glutamate and dopamine systems, and neuroendocrine systems such as the hypothalamic-pituitary-adrenal (HPA) axis, which are involved in sleep-wake-regulation and implicated in the pathogenesis of depression [4-6]. During states with increased proinflammatory cytokine production or after proinflammatory cytokine administration, humans and laboratory animals exhibit anhedonia, psychomotor slowing, sleepiness, fever and increased sensitivity to pain, termed 'sickness behavior' $[4,7]$. In depression, proinflammatory cytokines were found to interact with the HPA axis, to stimulate the serotonin-depleting indolamine-2,3-dioxygenase and to trigger serotonin reuptake $[6,8,9]$. Levels of proinflammatory cytokines were repeatedly found to be elevated in depressed patients as well as in animal models of depression $[6,10,11]$, potentially representing an inflammation-associated specific subtype of MDD [12-14] and a target for new antidepressant treatment strategies $[15,16]$.

Moreover, a body of studies applying objective and subjective measures for conditions of sleep and wakefulness, performed in both preclinical and clinical settings, showed that proinflammatory cytokines like tumor necrosis factor (TNF)- $\alpha$ and interferon (IFN)- $\gamma$ exhibit sleep-inducing properties. Somnogenic properties of proinflammatory cytokines, best researched for TNF- $\alpha$ and interleukin (IL)- $1 \beta$, presumably arise from activation of the humoral agents nuclear factor-kappa B (NF- $\kappa B$ ) and nitric oxide. Somnogenic effects may be further promoted by an impact on the HPA axis, including an increase in growth hormone-releasing hormone [5, 17-19]. States of low-grade inflammation with upregulation of cytokine signaling feature in disorders in which excessive daytime sleepiness is commonly observed, such as metabolic syndrome [20], obstructive sleep apnea and HLADR2-positive narcolepsy [21,22]. Cytokine levels are further related to certain habits like daily napping and the extent of physical activity [10]. Whereas the connection between sleep and cytokines has been more broadly investigated, little is known concerning the role of cytokines in arousal and wakefulness regulation. Predominately, anti-inflammatory cytokines such as IL-4, IL-10 and IL13 are regarded to have wakefulness-promoting effects. However, this was mostly concluded by the impact on sleep duration and sleep stage fractions in animal models $[17,18,23]$. In these, the administration of IL-10 and IL13 reduced time spent asleep, probably by limiting the production of sleep-inducing TNF- $\alpha$ and IL- 1 as well as interfering with transcription factor NF- $\kappa B$ [24-28]. Wake-promoting cytokines further peak in concentrations during daytime [29], levels of IL-10 reduce during sleep [30], sleep restriction leads to an increase in the IL-4 to IL-2 ratio [31] and insomniac men show higher levels of IL-4 than noninsomniac men [32].

In the present study, we used a recently developed and validated EEG-based algorithm which allows objective assessment of different EEG vigilance stages from active wakefulness to sleep onset. The aim of this study was to explore the relationship between EEG vigilance regulation and serum levels of wakefulness-promoting and somnogenic cytokines in depressed and nondepressed participants.

\section{Methods}

\section{Subjects}

The 189 participants comprised 35 consecutively admitted depressed patients from the Department of Psychiatry and Psychotherapy of the University Hospital Leipzig, 25 depressed patients from the outpatient clinic of the Integrated Research and Treatment Center for Adiposity Diseases Leipzig (IFB) and 54 nondepressed patients from the IFB and 75 community volunteers recruited via announcements (intranet, internet, local newspapers). A mix of obese and nonobese subjects was made possible given a missing relationship between EEG vigilance regulation and BMI 
(data not shown). Evaluation of the inclusion and exclusion criteria was performed in two stages.

Initial telephone screening comprised sociodemographic data, screening for somatic disorders and a checklist version of the Structured Clinical Interview for DSM-IV (SCID-I) [33]. Then, for both community subjects and patients treated at the clinics, a full SCID-I was performed in cases of positive SCID screening or known diagnosis of depression. Further, current and past history of mental and physical health problems and laboratory investigations for current inflammation were assessed.

Exclusion criteria were other DSM-IV axis 1 disorders, any psychoactive medication in the past 7 days, use of illegal drugs, alcohol abuse within the past 6 months, a history of head injury with loss of consciousness exceeding $1 \mathrm{~h}$, acute or chronic infection and major somatic disorders. Participants with known sleep apnea or obesity hypoventilation syndrome were also excluded.

A Hamilton Depression Rating Scale (HAMD-17) [34] was performed in depressed participants. All participants completed a Beck Depression Inventory (BDI-II) [35] and a sleep questionnaire ('Schlaffragebogen A'; SF-A) [36] assessing subjective sleep quality during the night preceding the EEG recording. The SF-A assesses five domains: 'sleep quality', 'feeling of being refreshed in the morning', 'psychosomatic complaints during the sleep phase', 'mental balance in the evening' and 'mental tiredness in the evening'. Written informed consent was obtained from all participants. The study was approved by Leipzig University Ethics Committee (No. 015-10-18012009).

\section{EEG Recordings}

Fifteen minutes of resting-state EEG were recorded. All recordings were taken between 08:00 and 18.00 h. Participants were instructed to keep their eyes closed, relax and not resist any urge to fall asleep. The EEG was recorded with a 40-channel QuickAmp amplifier (Brain Products GmbH, Gilching, Germany) from 31 electrode sites according to an extended version of the international 10-20 system at a sampling rate of $1 \mathrm{kHz}$, referenced against the common average using a low-pass filter at $280 \mathrm{~Hz}$. Impedances were kept below $10 \mathrm{k} \Omega$. EEG data were processed using a Brain Vision Analyzer 2.0 (Brain Products GmbH). EOG (electrooculogram) electrodes were placed above the upper left eye and under the lower right eye. EEG raw data were filtered at $70 \mathrm{~Hz}$ (low-pass), $0.5 \mathrm{~Hz}$ (high-pass) and $50 \mathrm{~Hz}$ (notch filter, range $\pm 2 \mathrm{~Hz}$ ). EOG channels were screened for periods of open eyes which were excluded from further analysis. Eye movement artifacts were removed with an independent component analysis approach by extracting 1-3 independent components that clearly represented vertical and horizontal eye movements. Likewise, persistent muscle artifacts were removed using the independent component analysis method. Following this, segments with remaining muscle, movement, eye and sweating artifacts were marked for exclusion from further analysis.

\section{Assessment and Classification of EEG Vigilance}

Each of the consecutive 900 one-second segments for each participant was classified according to the seven EEG vigilance stages of the computer-based VIGALL (Vigilance Algorithm Leipzig; http://www.uni-leipzig.de/ vigall/):

- Stage W with low-amplitude, non-alpha activity during an activated state

Cytokine Levels and EEG Vigilance

Regulation
- Stage A with dominant alpha activity corresponding to relaxed wakefulness (divided into substages A1, A2 and A3 according to the degree of spreading of alpha activity from occipital to more anterior cortices)

- Stage B with low-amplitude non-alpha (substage B1) and increasing theta and delta activity corresponding to drowsiness (substage B2/3)

- Stage C with sleep spindles or K-complexes characterizing sleep onset

Percentages of EEG vigilance stages were computed by dividing the number of segments of a given stage by the number of nonartifact segments [for a detailed description, see 3]. Furthermore, according to the respective vigilance stage, each segment was allocated to a measure ranging from the value 7 (highest vigilance stage $\mathrm{W}$ ) to 1 (sleep onset stage $\mathrm{C}$ ). The 15-min resting EEG was then separated into fifteen epochs with a duration of $1 \mathrm{~min}$ ( $\min 1, \min 2, \ldots, \min 15)$ and five epochs with a duration of $3 \mathrm{~min}$ (min $1-3$, min $4-6, \ldots$, min 13-15). Average vigilance values were computed for the respective epochs (online supplementary table; see www.karger.com/doi/10.1159/000441419).

\section{Cytokine Measurement}

All blood draws were performed immediately preceding the resting-state EEG recordings. Blood samples were immediately centrifuged at 3,000 rpm for $10 \mathrm{~min}$. The supernatant was aliquoted and stored in nonabsorbing polypropylene $300-\mu l$ tubes. Probes of serum were shock-frozen in liquid nitrogen and stored in freezers at $-80^{\circ} \mathrm{C}$ until further measurement. Cytokines were measured using the Bio-Plex Pro $^{\mathrm{TM}}$ human cytokine Th1/Th2 immunoassay from Bio-Rad, Germany, a 96-well kit that includes coupled magnetic beads and detection antibodies. This multiplex assay detects IL-2, IL-4, IL-5, IL-10, IL-12, IL-13, GM-CSF, IFN- $\gamma$ and TNF- $\alpha$. Levels of these cytokines and an evaluation of differences between depressed and nondepressed subjects in the present study sample have been reported [10]. In the present article, we aimed to explore a possible relationship between cytokines and vigilance regulation and therefore only included those subjects with EEG recordings matching the inclusion criteria.

\section{Statistics}

For differences in sociodemographic data and depression scores, Student's t test (age, BMI), $\chi^{2}$ test (sex) and Mann-Whitney $\mathrm{U}$ test (BDI-II) were performed. Of the 9 cytokines assayed, those with somnogenic properties (TNF- $\alpha$, IFN- $\gamma$, IL-2) and wakefulness-promoting properties (IL-4, IL-10, IL-13) were selected based on the literature [17]. Z-transformations were performed for each of these 6 individual cytokine levels and Z-values for TNF- $\alpha$, IFN- $\gamma$ and IL-2 were summed to produce the covariate 'somnogenic cytokines' and Z-values for IL-4, IL-10 and IL-13 to produce the covariate 'wakefulness-promoting cytokines'.

EEG vigilance stages were compared using the Mann-Whitney $U$ test due to a nonnormal distribution of stages, based on the Kolmogorov-Smirnov test. The average vigilance values for the fifteen 1-min and five 3-min epochs showed a normal distribution. The relationship between depression and the time course of EEG vigilance was examined in a 2 ('group': depressed vs. nondepressed) $\times 5$ ('time': $\min 1-3, \min 4-6, \min 7-9$, min 10-12, min 13-15 epochs) repeated measures ANOVA, with the average vigilance value per 3-min epoch as the dependent variable and time of recording as the covariate. Post hoc tests for the five 


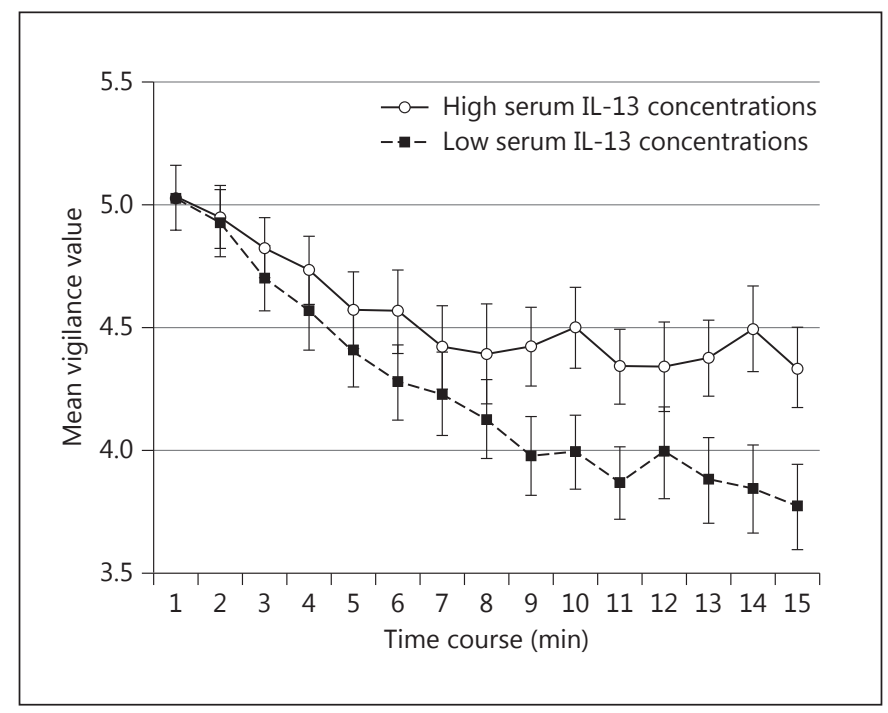

Fig. 1. Time course of EEG vigilance between groups of participants with 'high IL-13' (4th quartile $=$ IL-13 $\geq 8.44 \mathrm{pg} / \mathrm{ml}, \mathrm{n}=47$ ) and 'low IL-13' (1st quartile = IL-13 $\leq 2.48 \mathrm{pg} / \mathrm{ml}, \mathrm{n}=49$ ).

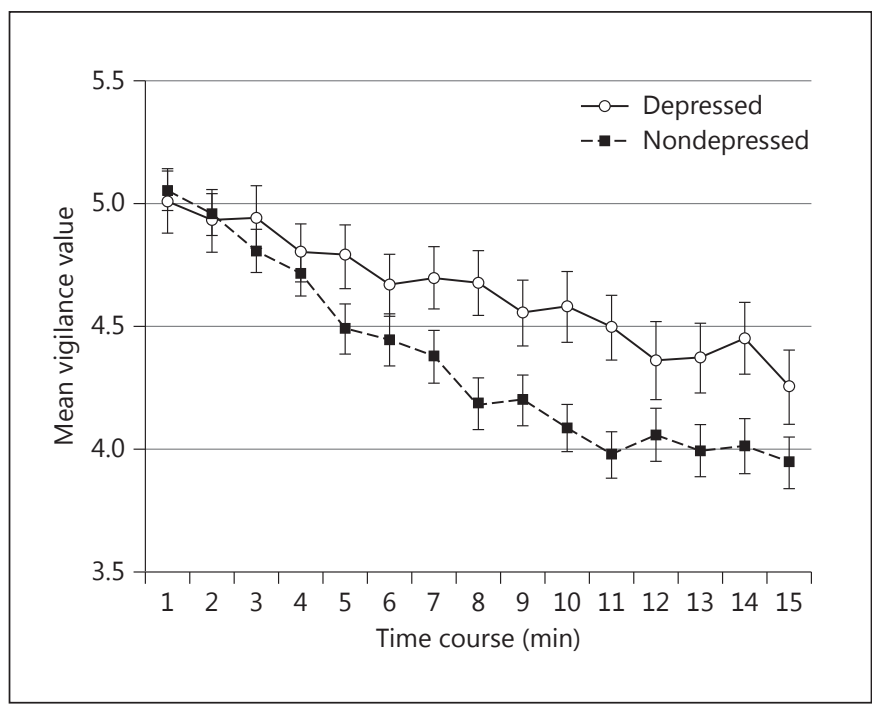

Fig. 2. Time course of EEG vigilance between groups of depressed and nondepressed participants.

Table 1. Differences in sociodemographics, depression scores, time of EEG recordings and sleep quality between groups of depressed and nondepressed participants

\begin{tabular}{|c|c|c|c|c|}
\hline & $\begin{array}{l}\text { Depressed } \\
\text { participants }(n=60)\end{array}$ & $\begin{array}{l}\text { Nondepressed } \\
\text { participants }(n=129)\end{array}$ & Test statistics & $\mathrm{p}$ values \\
\hline Age, years & $39.30 \pm 13.02$ & $37.12 \pm 12.85$ & $\mathrm{~T}=-1.074$ & 0.285 \\
\hline BMI & $29.61 \pm 9.70$ & $36.24 \pm 12.11$ & $\widehat{\mathrm{T}}=1.011$ & 0.314 \\
\hline BDI-II score & $27.19 \pm 11.68$ & $6.18 \pm 6.69$ & $Z=-9.702$ & $<0.001$ \\
\hline HAMD-17 score & $17.56 \pm 6.65$ & - & n.a. & n.a. \\
\hline Sleep quality (SF-A) & $2.67 \pm 0.80$ & $3.81 \pm 0.67$ & $Z=-7.938$ & $<0.001$ \\
\hline Refreshed in the morning (SF-A) & $2.69 \pm 0.90$ & $3.33 \pm 0.65$ & $Z=-4.421$ & $<0.001$ \\
\hline Mental balance in the evening (SF-A) & $2.27 \pm 0.82$ & $3.46 \pm 0.71$ & $Z=-7.506$ & $<0.001$ \\
\hline Mental tiredness in the evening (SF-A) & $3.48 \pm 0.96$ & $2.94 \pm 0.83$ & $Z=-3.742$ & $<0.001$ \\
\hline Psychosomatic complaints during sleep (SF-A) & $1.73 \pm 0.70$ & $1.33 \pm 0.37$ & $Z=-3.532$ & $<0.001$ \\
\hline Self-reported sleep duration, $\mathrm{h} / \mathrm{min}$ & $7 / 47 \pm 1 / 73$ & $7 / 18 \pm 1 / 17$ & $Z=-1.278$ & 0.201 \\
\hline
\end{tabular}

Data are presented as means \pm SD unless otherwise indicated. Significant comparisons are in italics. n.a. $=$ Not applicable.

${ }^{1} \mathrm{SD}$ indicates hours and minutes.

3-min blocks were performed with Scheffé tests with time of recording as the covariate.

Analyses of covariance with repeated measures (MANCOVA) were used to investigate the relationships between depression, cytokine levels and the time course of EEG vigilance. In a first step, separate MANCOVAs were performed in the total sample and the two subgroups (depressed vs. nondepressed participants; fig. 1), with the average vigilance value serving as the dependent variable and 'time' serving as the within-subject factor, controlled for levels of each separate cytokine or levels of somnogenic or wakefulness-promoting cytokines (covariate). Two separate 2 (between-subject factor 'group') $\times 5$ (repeated measure 'time') MANCOVAs were then performed in the total sample, with levels of somnogenic or wakefulness-promoting cytokines as the respective covariate and time of recording as the covariate. For figure 2 , the subjects were allocated into groups of 'high IL-13' and 'low IL-13' when levels were below the 1st quartile (IL-13 $\leq 2.48 \mathrm{pg} / \mathrm{ml}, \mathrm{n}=49$ ) or above the 4 th quartile (IL-13 $\geq 8.44 \mathrm{pg} / \mathrm{ml}, \mathrm{n}=47$ ). The IBM Statistical Package for the Social Sciences (SPSS) program version 20.0 for Windows was used for all statistical analyses. The significance level was set at $\mathrm{p}<0.05$. 


\section{Results}

The depressed and nondepressed groups did not differ in age, sex or BMI. As expected, the depressed participants showed significantly higher self-rated scores on the BDI-II, with overall moderate severity of depressive symptoms according to HAMD-17 (table 1). On the SF-A, the depressed participants reported reduced 'sleep quality', reduced 'feeling of being refreshed in the morning', increased 'psychosomatic complaints during the sleep phase', reduced 'mental balance in the evening' and increased 'mental tiredness in the evening'. Reported sleep duration did not vary between groups. The EEG recording was performed slightly later in the day in the nondepressed group (table 1).

\section{EEG Vigilance and Cytokines}

Repeated measures ANOVA analyses of EEG vigilance by time for each of the cytokines revealed significance for the factor 'IL-13' in the total sample, and an 'IL-13 $\times$ time' interaction in the total sample and in both depressed patients and controls (fig. 1). For grouped cytokines, a significant interaction for 'wakefulness-promoting cytokines $\times$ time' was found within the total sample. Within the depressed group, there was a significant interaction 'wakefulness-promoting cytokines $\times$ time'. Within the nondepressed controls no interaction 'wakefulness-promoting cytokines $\times$ time' could be found. No significant interaction for 'somnogenic cytokines $\times$ time' was found within the total sample or separate groups (table 2).

\section{EEG Vigilance in Depression}

Comparisons of EEG vigilance stages between groups revealed a significantly higher amount of total A stages and substage A2 and a lower amount of total B stages in the depressed compared to nondepressed groups (table 3). Repeated measures ANOVA of EEG vigilance course between groups revealed a highly significant effect of the factor 'group' on the dependent variable 'average vigilance value'. The interaction 'group $\times$ time' was also significant (table 3 ). Post hoc tests revealed significant group differences for the min 7-9, min 10-12 and min 13-15 epochs (fig. 2).

\section{Impact of Cytokine Levels on EEG Vigilance in \\ Depression}

ANOVA analysis of the dependent variable 'average vigilance value' between groups showed significant interactions in 'group $\times$ time' $\left(\mathrm{F}_{2,444}=2.995, \mathrm{p}=0.041\right)$ and in

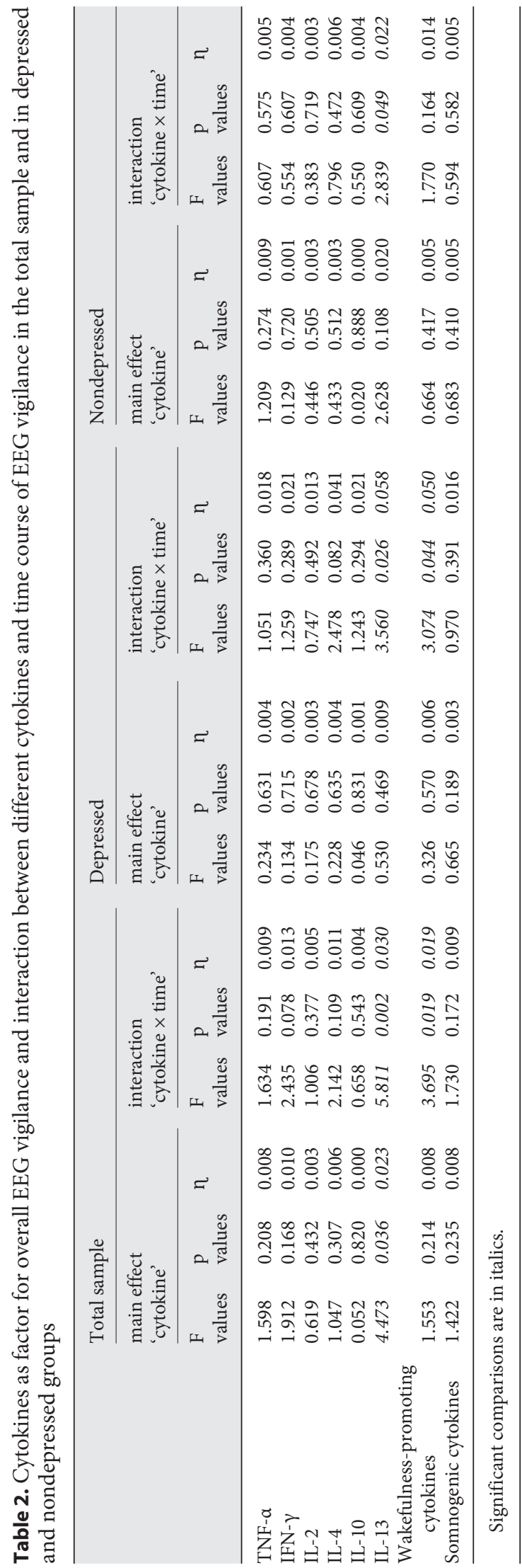

Cytokine Levels and EEG Vigilance Regulation
Neuropsychobiology 2016;73:1-9 DOI: $10.1159 / 000441190$ 
Table 3. Occurrence of EEG vigilance stages between depressed and nondepressed groups and differences of EEG vigilance regulation over time

\begin{tabular}{|c|c|c|c|c|c|}
\hline & $\begin{array}{l}\text { Depressed } \\
\text { value }\end{array}$ & $\begin{array}{l}\text { Nondepressed } \\
\text { value }\end{array}$ & Test statistics & $\mathrm{p}$ values & Partial $\eta^{2}$ \\
\hline \multicolumn{6}{|l|}{ Stage, $\%$} \\
\hline Stage W & $12.98 \pm 16.57$ & $12.50 \pm 15.79$ & $\mathrm{~F}=0.036$ & 0.849 & 0.000 \\
\hline Stage A & $52.62 \pm 30.08$ & $42.83 \pm 28.65$ & $F=4.636$ & 0.033 & 0.024 \\
\hline Substage A1 & $22.61 \pm 23.07$ & $21.38 \pm 21.61$ & $\mathrm{~F}=0.126$ & 0.723 & 0.001 \\
\hline Substage A2 & $27.26 \pm 24.95$ & $17.99 \pm 19.79$ & $F=7.572$ & 0.007 & 0.039 \\
\hline Substage A3 & $2.76 \pm 3.63$ & $3.45 \pm 5.76$ & $\mathrm{~F}=0.763$ & 0.383 & 0.004 \\
\hline Stage B & $34.08 \pm 26.72$ & $43.51 \pm 25.15$ & $F=5.533$ & 0.020 & 0.029 \\
\hline Substage B1 & $21.32 \pm 20.98$ & $27.06 \pm 21.10$ & $\mathrm{~F}=3.039$ & 0.083 & 0.016 \\
\hline Substage B2/3 & $12.76 \pm 17.59$ & $16.45 \pm 18.07$ & $\mathrm{~F}=1.739$ & 0.189 & 0.009 \\
\hline Stage C & $0.32 \pm 1.30$ & $1.16 \pm 4.33$ & $\mathrm{~F}=2.164$ & 0.143 & 0.011 \\
\hline \multicolumn{6}{|c|}{ Repeated measures ANOVA } \\
\hline Time & & & $\mathrm{F}=1.423$ & 0.240 & 0.008 \\
\hline Group & & & $F=3.929$ & 0.049 & 0.021 \\
\hline Time $\times$ group & & & $F=3.424$ & 0.026 & 0.018 \\
\hline Post hoc min $1-3$ & $4.96 \pm 0.94$ & $4.93 \pm 0.87$ & $\mathrm{~F}=0.044$ & 0.834 & 0.000 \\
\hline Post hoc min $4-6$ & $4.75 \pm 0.92$ & $4.55 \pm 1.06$ & $\mathrm{~F}=1.632$ & 0.203 & 0.009 \\
\hline Post hoc min 7-9 & $4.64 \pm 0.93$ & $4.25 \pm 1.12$ & $F=5.036$ & 0.026 & 0.026 \\
\hline Post hoc $\min 10-12$ & $4.48 \pm 1.06$ & $4.04 \pm 1.03$ & $F=6.536$ & 0.011 & 0.034 \\
\hline Post hoc $\min 13-15$ & $4.36 \pm 1.03$ & $3.98 \pm 1.12$ & $F=4.579$ & 0.034 & 0.024 \\
\hline
\end{tabular}

Data are presented as means \pm SD unless otherwise indicated. Significant comparisons are in italics.

'wakefulness-promoting cytokines $\times$ time' $\left(\mathrm{F}_{2,444}=3.105\right.$, $\mathrm{p}=0.037)$. 'EEG vigilance' trended for the factor 'group' $\left(\mathrm{F}_{1,185}=3.416, \mathrm{p}=0.066\right)$ but not for 'wakefulness-promoting cytokines' $\left(\mathrm{F}_{1,185}=0.968, \mathrm{p}=0.326\right)$.

\section{Discussion}

This is, to the authors' knowledge, the first study comparing resting-state EEG measures and cytokine serum concentrations in unmedicated depressed and nondepressed participants. The results demonstrate a positive association between EEG-based measures of brain arousal (EEG vigilance) and concentrations of cytokines in the total sample and separate groups, which was not related to differences in brain arousal regulation between MDD and nondepressed controls.

This positive association was not a general finding for all 6 cytokines investigated but limited to IL- 13 and, possibly driven by IL-13, the grouped IL-4, IL-10 and IL-13 cytokines with wakefulness-regulating properties. For the individual and summed somnogenic cytokines TNF- $\alpha$, IFN- $\gamma$ and IL-2, all mediators of proinflammatory immune responses, no such association could be shown. Notwithstanding that proinflammatory cytokines such as TNF- $\alpha$ have been most often implicated in the pathophysiology of depression [12, 37], our results indicate that anti-inflammatory, wakefulness-promoting cytokines such as IL-4, IL-10 and IL-13 should be borne in mind when investigating specific features of depression such as abnormalities in sleep-wake regulation. Both the anti-inflammatory and wakefulness-promoting properties of IL-13, levels of which are reported to be elevated in MDD [10], potentially arise from the suppression of important molecular pathway agents like second messenger nitric oxide release [38] and, together with IL- 4 and IL-10, by the blockade of the transcriptional factor NF- $\kappa \mathrm{B}$ [5]. Indirect sleep-inhibiting effects of these cytokines may also arise from the inhibition of the differentiation and shift of naïve T cells into T-helper 1 cells and activation of macrophages, respectively, from a reduction of TNF- $\alpha$ and IFN- $\gamma$ levels. Peripheral and hypothalamic TNF- $\alpha$ has been suggested to show a direct impact on non-REM sleep and modulate molecular networks relevant for sleep regulation $[5,17,19]$. TNF- $\alpha$ levels were linked with daytime sleepiness [39] and levels increased after supplementation with sleep-inducing antidepressants and antipsychotics [40, 41]. Whilst IFN- $\gamma$ - 
mediated sleep-inducing properties are considered to depend on the interaction with TNF- $\alpha$, as deduced from symptoms of sleepiness and fatigue during infections and IFN treatments $[5,17]$, data is lacking on its role on wakefulness. Several lines of evidence link the immune response to arousal regulation, possibly via the HPA axis or monoaminergic transmitter systems $[1,4,5]$. Subjects suffering from inflammation frequently exhibit tiredness or sleepiness mediated by the increased production of proinflammatory cytokines in the setting of 'sickness behavior', which resembles depressive symptoms [4, 7]. Narcolepsy, a disorder with increased daytime sleepiness, was found to be associated with specific markers of the HLA system and increased production of proinflammatory cytokines $[21,42,43]$. Cytokines not only influence wakefulness regulation, but their concentrations also change depending on sleep characteristics or following sleep deprivation [44-46].

Our finding of more stable EEG vigilance regulation in MDD is in agreement with previous studies $[2,3]$ and extends past findings using a refined version of the VIGALL algorithm in a larger sample of unmedicated depressed patients. The pattern across the 15-min EEG sampling frame of fewer declines into lower EEG vigilance stages, and a greater percentage of high EEG vigilance stages in the unmedicated depressed group, supports the theory of hyperstable arousal regulation in MDD [1]. This potential pathophysiological feature of depression is considered to be a state-dependent trait, with depressive symptoms both as features of the hyperstable brain arousal regulation (e.g. sleep disturbances, diurnal variation of mood) and as a result of an autoregulation response to this (e.g. social withdrawal, sensation avoidance). Findings of hyperstable brain arousal regulation are not exclusive to MDD, having been recently demonstrated in obsessive-compulsive disorder [47]. This pathophysiological overlap between the disorders may contribute to high reciprocal comorbidity rates [48]. Our results of diminished sleep quality in depressed patients is also in accordance with the literature [49] and these sleep disturbances may be a result of hyperstable wakefulness regulation not allowing patients to reach lower arousal levels that would be needed to attain sleep or deep sleep.

Despite the interactions found between cytokines and EEG vigilance herein as well as both altered brain arousal regulation and cytokine levels in MDD, cytokines did not significantly influence EEG vigilance differences between the groups. Therefore, our findings do not provide support for a pathogenic link between hyperstable brain arousal regulation and proinflammato-

Cytokine Levels and EEG Vigilance

Regulation ry/somnogenic or anti-inflammatory/wakefulness-promoting cytokines.

There are several limitations of this study. As well as analyzing the impact of each cytokine on EEG vigilance regulation separately, we grouped cytokines into wakefulness-promoting and somnogenic cytokines based on a review of the published literature [17]. This is a somewhat imprecise classification, grouping cytokines from results of experimentally and statistically heterogeneous previous studies. Another limitation is that both blood samples and EEG measurements were taken at varying hours of the day, between 8:00 and 18:00 h. Therefore, an influence of circadian rhythmicity on the levels and effects of cytokines cannot be excluded $[50,51]$. The temporal resolution of EEG and cytokine measures and the extent to which they may be linked to changes in brain arousal are also disparate. Whereas the EEG has the methodological strength of instant and objective vigilance recording, changes in peripheral cytokines show latency due to synthesis and secretion and the passing of the blood-brain barrier [4]. Although not investigated to date, measurable changes in cytokine concentrations related to short-term, nonrecurring changes in EEG vigilance can hardly be expected, whereas a longterm recording with, for example, sustained high vigilance regulation, prolonged vigilance patterns could be accompanied by sustained changes in cytokine concentrations. Therefore, the methods applied in the present study may account for not finding a link between somnogenic cytokines and arousal patterns. The 15-min EEG recording time during which the vast majority of participants did not manifest signs of sleep onset may have been too brief to elicit or discern neurophysiological connections between somnogenic cytokines and parameters of sleep onset. However, as most previous studies used subjective questionnaires to assess sleepiness or wakefulness while investigating the relationship between cytokines and wakefulness regulation [17] and as this is the first study investigating serum cytokine levels together with EEG measurements combined with VIGALL, we think that this negative result is important to report.

In conclusion, this study provides further evidence for hyperstable brain arousal regulation in depressed patients and for a putative role of the immune system in wakefulness regulation. The hyperstable brain arousal regulation in MDD and elevated cytokine levels in this disorder appear to be the most likely independent pathways relevant to the pathogenesis of MDD, a subject requiring further investigation. The observed connection

Neuropsychobiology 2016;73:1-9

DOI: $10.1159 / 000441190$ 
between serum levels of cytokines and objective, algorithm-based, measurement of EEG vigilance indicates the potential utility of studies combining EEG vigilance and EEG sleep measures, along with repeated cytokine assaying during the recordings, to elucidate this interesting but little-investigated field of psychoneuroimmunology.

\section{Acknowledgments}

We thank Dr. Roland Mergl for statistical assistance and Wolfgang Wilfert for technical support. This work was financially supported by the Federal Ministry of Education and Research (BMBF), Germany (FKZ: 01EO1001) and the Claussen-Simon Foundation.

\section{Disclosure Statement}

Prof. Himmerich received speaker honoraria from AstraZeneca, Lilly and Servier, consulting fees from Bristol-Myers Squibb and chemical substances for study support from AstraZeneca, Novartis and Wyeth. Within the last 3 years, Prof. Hegerl was an advisory board member for Lilly, Lundbeck, Takeda Pharmaceuticals and Otsuka Pharma, a consultant for Nycomed and a speaker for Bristol-Myers Squibb, MEDICE Arzneimittel and Roche Pharma. All other authors report no conflicts of interest.

\section{References}

1 Hegerl U, Hensch T: The vigilance regulation model of affective disorders and ADHD. Neurosci Biobehav Rev 2014;44:45-57.

2 Hegerl U, Wilk K, Olbrich S, Schoenknecht P, Sander C: Hyperstable regulation of vigilance in patients with major depressive disorder. World J Biol Psychiatry 2012;13:436-446.

3 Olbrich S, Sander C, Minkwitz J, Chittka T, Mergl R, Hegerl U, Himmerich H: EEG vigilance regulation patterns and their discriminative power to separate patients with major depression from healthy controls. Neuropsychobiology 2012;65:188-194.

4 Capuron L, Miller AH: Immune system to brain signaling: neuropsychopharmacological implications. Pharmacol Ther 2011;130: 226-238.

5 Krueger JM, Majde JA, Rector DM: Cytokines in immune function and sleep regulation. Handb Clin Neurol 2011;98:229-240.

6 Himmerich H, Binder EB, Kunzel HE, Schuld A, Lucae S, Uhr M, Pollmacher T, Holsboer F, Ising M: Successful antidepressant therapy restores the disturbed interplay between TNF- $\alpha$ system and HPA axis. Biol Psychiatry 2006;60:882-888.

7 Dantzer R: Cytokine-induced sickness behavior: mechanisms and implications. Ann NY Acad Sci 2001;933:222-234.

8 Muller N, Schwarz MJ: The immune-mediated alteration of serotonin and glutamate: towards an integrated view of depression. Mol Psychiatry 2007;12:988-1000.

9 Malynn S, Campos-Torres A, Moynagh P, Haase J: The pro-inflammatory cytokine TNF- $\alpha$ regulates the activity and expression of the serotonin transporter (SERT) in astrocytes. Neurochem Res 2013;38:694-704.

10 Schmidt FM, Lichtblau N, Minkwitz J, Chittka T, Thormann J, Kirkby KC, Sander C, Mergl R, Fasshauer M, Stumvoll M, Holdt LM,
Teupser D, Hegerl U, Himmerich H: Cytokine levels in depressed and non-depressed subjects, and masking effects of obesity. J Psychiatr Res 2014;55:29-34.

11 Krugel U, Fischer J, Radicke S, Sack U, Himmerich H: Antidepressant effects of TNF- $\alpha$ blockade in an animal model of depression. J Psychiatr Res 2013;47:611-616.

12 Lichtblau N, Schmidt FM, Schumann R, Kirkby $\mathrm{KC}$, Himmerich $\mathrm{H}$ : Cytokines as biomarkers in depressive disorder: current standing and prospects. Int Rev Psychiatry 2013;25: 592-603.

13 Felger JC, Lotrich FE: Inflammatory cytokines in depression: neurobiological mechanisms and therapeutic implications. Neuroscience 2013;246:199-229.

14 Lotrich FE: Inflammatory cytokine-associated depression. Brain Res 2015 18;1617:113125.

15 Noto C, Rizzo LB, Mansur RB, McIntyre RS, Maes M, Brietzke E: Targeting the inflammatory pathway as a therapeutic tool for major depression. Neuroimmunomodulation 2014; 21:131-139.

16 Schmidt FM, Kirkby KC, Himmerich H: The TNF- $\alpha$ inhibitor etanercept as monotherapy in treatment-resistant depression - report of two cases. Psychiatr Danub 2014;26:288-290.

17 Weschenfelder J, Sander C, Kluge M, Kirkby $\mathrm{KC}$, Himmerich $\mathrm{H}$ : The influence of cytokines on wakefulness regulation: clinical relevance, mechanisms and methodological problems. Psychiatr Danub 2012;24:112-126.

18 Krueger JM, Majde JA: Humoral links between sleep and the immune system: research issues. Ann NY Acad Sci 2003;992:9-20.

19 Kapsimalis F, Basta M, Varouchakis G, Gourgoulianis K, Vgontzas A, Kryger M: Cytokines and pathological sleep. Sleep Med 2008; 9:603-614.
20 Schmidt FM, Weschenfelder J, Sander C, Minkwitz J, Thormann J, Chittka T, Mergl R, Kirkby KC, Fasshauer M, Stumvoll M, Holdt LM, Teupser D, Hegerl U, Himmerich H: Inflammatory cytokines in general and central obesity and modulating effects of physical activity. PLoS One 2015;10:e0121971.

21 Himmerich H, Beitinger PA, Fulda S, Wehrle R, Linseisen J, Wolfram G, Himmerich S, Gedrich K, Wetter TC, Pollmacher T: Plasma levels of tumor necrosis factor alpha and soluble tumor necrosis factor receptors in patients with narcolepsy. Arch Intern Med 2006; 166:1739-1743.

22 Zhan S, Cai G, Zheng A, Wang Y, Jia J, Fang $\mathrm{H}$, Yang $\mathrm{Y}, \mathrm{Hu} \mathrm{M}$, Ding Q: Tumor necrosis factor-alpha regulates the Hypocretin system via mRNA degradation and ubiquitination. Biochim Biophys Acta 2011;1812:565-571.

23 Kapsimalis F, Richardson G, Opp MR, Kryger M: Cytokines and normal sleep. Curr Opin Pulm Med 2005;11:481-484.

24 Toth LA, Opp MR: Cytokine- and microbially induced sleep responses of interleukin-10 deficient mice. Am J Physiol Regul Integr Comp Physiol 2001;280:R1806-R1814.

25 Kushikata T, Fang J, Krueger JM: Interleukin-10 inhibits spontaneous sleep in rabbits. J Interferon Cytokine Res 1999;19:1025-1030.

26 Kubota T, Fang J, Kushikata T, Krueger JM: Interleukin-13 and transforming growth factor- $\beta 1$ inhibit spontaneous sleep in rabbits. Am J Physiol Regul Integr Comp Physiol 2000;279:R786-R792.

27 Kushikata T, Fang J, Wang Y, Krueger JM: Interleukin-4 inhibits spontaneous sleep in rabbits. Am J Physiol 1998;275:R1185-R1191.

28 Chen Z, Gardi J, Kushikata T, Fang J, Krueger JM: Nuclear factor- $\kappa B$-like activity increases in murine cerebral cortex after sleep deprivation. Am J Physiol 1999;276:R1812-R1818. 
29 Lange T, Dimitrov S, Born J: Effects of sleep and circadian rhythm on the human immune system. Ann NY Acad Sci 2010;1193:48-59.

30 Lange T, Dimitrov S, Fehm H, Westermann J, Born J: Shift of monocyte function toward cellular immunity during sleep. Arch Intern Med 2006;166:1695-1700.

31 Axelsson J, Rehman J, Akerstedt T, Ekman R, Miller GE, Hoglund CO, Lekander M: Effects of sustained sleep restriction on mitogenstimulated cytokines, chemokines and $\mathrm{T}$ helper 1/T helper 2 balance in humans. PLoS One 2013;8:e82291.

32 Sakami S, Ishikawa T, Kawakami N, Haratani T, Fukui A, Kobayashi F, Fujita O, Araki S, Kawamura N: Coemergence of insomnia and a shift in the Th1/Th2 balance toward Th2 dominance. Neuroimmunomodulation 2002-2003; 10:337-343.

33 Wittchen H-U, Zaudig M, Fydrich T: SKID Strukturiertes Klinisches Interview für DSMIV (Achse I und II). Göttingen, Hogrefe, 1997.

34 Hamilton M: A rating scale for depression. J Neurol Neurosurg Psychiatry 1960;23:56-62.

35 Beck AT, Steer RA, Brown GK: BDI-II, Beck Depression Inventory: Manual, ed 2. Boston, Harcourt Brace, 1996.

36 Görtelmeyer R: SF-A und SF-B. Schlaf-Fragebogen A und B; in Collegium Internationale Psychiatrie Scalarum (eds): Internationale Skalen für Psychiatrie, ed 4. Göttingen, Belz, 1996.

37 Berthold-Losleben $\mathrm{M}$, Himmerich $\mathrm{H}$ : The TNF- $\alpha$ system: functional aspects in depression, narcolepsy and psychopharmacology. Curr Neuropharmacol 2008;6:193-202.
38 Van Dyken, Steven J, Locksley RM: Interleukin-4- and interleukin-13-mediated alternatively activated macrophages: roles in homeostasis and disease. Annu Rev Immunol 2013; 31:317-343

39 Heesen C, Nawrath L, Reich C, Bauer N, Schulz K, Gold SM: Fatigue in multiple sclerosis: an example of cytokine mediated sickness behaviour? J Neurol Neurosurg Psychiatry 2006;77:34-39.

40 Himmerich H, Schonherr J, Fulda S, Sheldrick AJ, Bauer K, Sack U: Impact of antipsychotics on cytokine production in vitro. J Psychiatr Res 2011;45:1358-1365.

41 Petersein C, Sack U, Mergl R, Schönherr J, Schmidt FM, Lichtblau N, Kirkby KC, Bauer $\mathrm{K}$, Himmerich $\mathrm{H}$ : Impact of lithium alone and in combination with antidepressants on cytokine production in vitro. J Neural Transm 2015;122:109-122.

42 Fontana A, Gast H, Reith W, Recher M, Birchler T, Bassetti CL: Narcolepsy: autoimmunity, effector $\mathrm{T}$ cell activation due to infection, or T cell independent, major histocompatibility complex class II induced neuronal loss? Brain 2010;133:1300-1311.

43 Maurovich-Horvat E, Keckeis M, Lattova Z Kemlink D, Wetter T, Schuld A, Sonka K, Pollmacher T: Hypothalamo-pituitary-adrenal axis, glucose metabolism and TNF- $\alpha$ in narcolepsy. J Sleep Res 2014;23:425-431.

44 Besedovsky L, Lange T, Born J: Sleep and immune function. Pflugers Arch 2012;463:121137.
45 Vgontzas AN, Zoumakis E, Lin H, Bixler EO, Trakada G, Chrousos GP: Marked decrease in sleepiness in patients with sleep apnea by etanercept, a tumor necrosis factor- $\alpha$ antagonist. J Clin Endocrinol Metab 2004;89:44094413.

46 Voderholzer U, Fiebich BL, Dersch R, Feige B, Piosczyk H, Kopasz M, Riemann D, Lieb K: Effects of sleep deprivation on nocturnal cytokine concentrations in depressed patients and healthy control subjects. J Neuropsychiatry Clin Neurosci 2012;24:354-366.

47 Olbrich S, Olbrich H, Jahn I, Sander C, Adamaszek M, Hegerl U, Reque F, Stengler K: EEG-vigilance regulation during the resting state in obsessive-compulsive disorder. Clin Neurophysiol 2013;124:497-502.

48 Murphy DL, Moya PR, Fox MA, Rubenstein LM, Wendland JR, Timpano KR: Anxiety and affective disorder comorbidity related to serotonin and other neurotransmitter systems: obsessive-compulsive disorder as an example of overlapping clinical and genetic heterogeneity. Philos Trans R Soc Lond B Biol Sci 2013;368:20120435.

49 Harvey AG: Sleep and circadian functioning critical mechanisms in the mood disorders? Annu Rev Clin Psychol 2011;7:297-319.

50 Petrovsky N, McNair P, Harrison LC: Diurnal rhythms of pro-inflammatory cytokines: regulation by plasma cortisol and therapeutic implications. Cytokine 1998;10:307-312.

51 Lissoni P, Rovelli F, Brivio F, Brivio O, Fumagalli L: Circadian secretions of IL-2, IL-12, IL- 6 and IL-10 in relation to the light/dark rhythm of the pineal hormone melatonin in healthy humans. Nat Immun 1998;16:1-5. 